http://dx.doi.org/10.35381/e.k.v3i5.526

\title{
Andragogía en los estudios de postgrado. Una perspectiva teórica dialógica fenomenológica
}

\section{Andragogy in postgraduate studies. A theoretical phenomenological dialogic perspective}

\author{
Carlina Leonor García-Oberto \\ carlunermb@gmail.com \\ Universidad Nacional Experimental Francisco de Miranda, Santa Ana de Coro \\ Venezuela \\ https//orcid.org/0000-0002-3497-9187
}

Recepción: 01 Junio 2019

Revisado: 15 Julio 2019

Aprobación: 04 Agosto 2019

Publicación: 01 Enero 2020

\section{RESUMEN}

La investigación tuvo como propósito la comprensión de la praxis andragógica en los estudios de postgrado desde una perspectiva teórica dialógica y fenomenológica Husserliana. Se llevaron a cabo encuentros con 5 informantes clave quienes habían empleado el enfoque dialógico interactivo de forma apropiada. En tal sentido, la praxis andragógica queda definida como una acción innovadora por su constante adaptación a los cambios. También se considera trascendental, por cuanto el conocimiento se supera a sí mismo; además, es especializada debido a que atiende a distintos campos y niveles educativos. A manera de reflexión, se pudo concluir que el enfoque dialógico promueve no sólo la valoración de las herramientas tecnológicas sino también la formación de los individuos, ya que permite lograr la trascendencia en el intercambio de saberes tanto dentro de la universidad como fuera de ella.

Palabras Claves: Educación de adultos; personal académico docente; estudianteprofesor; educador de adultos. (Palabras tomadas del Tesauro UNESCO).

\begin{abstract}
The purpose of this research was to understand the andragogical praxis in postgraduate studies from a Husserlian dialogic and phenomenological theoretical perspective. Meetings were held with 5 key informants who had used the interactive dialogic
\end{abstract}


approach appropriately. In this sense, andragogic praxis is defined as an innovative action for its constant adaptation to changes. It is considered transcendental because knowledge exceeds itself, and also specialized because it serves different fields and educational levels. As a reflection, it may be concluded that the dialogic approach promotes not only the valuation of technological tools but also the training of individuals, since it allows achieving transcendence in the exchange of knowledge both inside and outside the university.

Keywords: Adult education; academic teaching personnel; student-teachers; adult educators. (Words taken from UNESCO Thesaurus).

\section{INTRODUCCIÓN}

En este primer momento hago una descripción de la situación que se vivencia dentro del contexto de la cosa misma, señalo los propósitos a abordar, destaco el aporte que éste forja en base a la generación de un constructo teórico; también, especifico la línea en la cual se adscribe el estudio y los aspectos que se enmarcan dentro de la perspectiva geográfica, teórica, temporal y metodológica. En este sentido, para cumplir con todos los apartados de un trabajo doctoral, me es necesario aclarar que para la titulación de cada capítulo, empleo un lenguaje fundamentado en las lecturas realizadas de los escritos fenomenológicos de Husserl, cuya concepción constituye la naturaleza primordial del camino investigativo a recorrer. Por ello, denominé este momento "El escenario en su esencia" e inicio con el planteamiento de la situación en términos de "La realidad en su esencia".

\section{La realidad en su esencia}

La fenomenología constituye una vertiente significativa dentro del campo investigativo; por ello, puede ser considerada como una vía para llevar a cabo estudios relacionados con vivencias que requieran de un análisis profundo bajo las argumentaciones de sus propios protagonistas. Por ende, es menester considerar dicha vía desde la perspectiva trascendental del padre de la fenomenología, es decir, de Husserl, quien aunque 


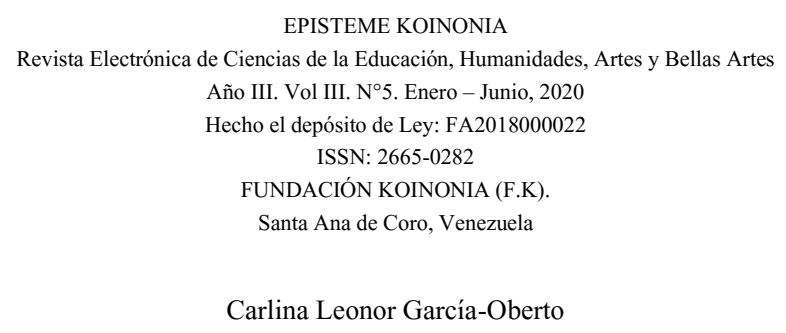

presenta ciertas críticas por su afán de llegar a la conciencia del ser, aportó una teoría capaz de orientar la comprensión del ser y su criterio judicativo sobre la esencia de las cosas.

De este modo, Husserl (1949) nos conduce a concretar el significado del ser desde un punto de vista fenomenológico, en el que citando palabras propias del mismo, declara lo siguiente: "la fenomenología pura o trascendental se funda "no en una ciencia de hechos sino como una ciencia de esencias (como una ciencia eidética), como una ciencia que quiere llegar exclusivamente a "conocimientos esenciales" y no fijar, en absoluto, "hechos" (p. 8).

Al respecto, emerge mi idea de abordar una realidad educativa que discurre por la visión propia del sujeto quien al ser partícipe de un proceso andragógico mediado por un enfoque dialógico interactivo, ostenta la esencia de la cosa misma. Este enfoque constituye un estilo de formación fundamentado en el diálogo y la interacción a través dos modalidades (presencial y virtual), las cuales propician la comunicación constante y el aprendizaje continuo y permanente.

Atendiendo a lo anterior, para el año 2002, en el Estado Falcón, específicamente, en la Universidad Nacional Experimental "Francisco de Miranda" se crea una modalidad que abre las puertas tanto a estudiantes sin ocupación como a personas que trabajan para brindar una oportunidad de estudio de pregrado con características de dialogicidad e interacción mediados por el uso de las tecnologías de información y comunicación (TIC).

Para el año 2012, inicia la propuesta para elaborar aulas virtuales que sirvieran de soporte tecnológico a los estudios de postgrados en el Área de Educación. Para tal fin, los docentes tuvieron la oportunidad de participar en una jornada de formación, donde recibieron una orientación fundamentada en el aprendizaje dialógico interactivo y elaboraron los diseños instruccionales de cada asignatura. En dicha jornada se logró la 


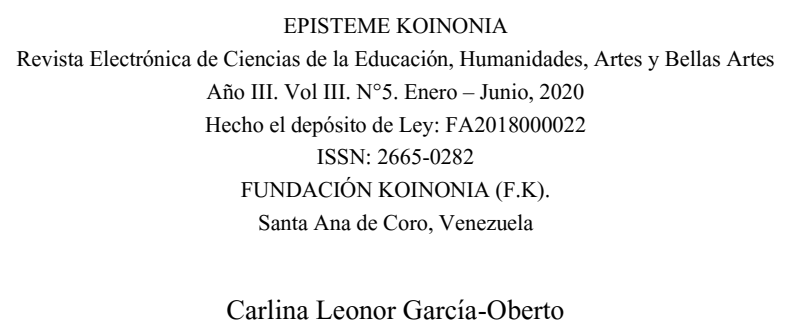

elaboración de las aulas virtuales con sus respectivos recursos y estrategias; así como la elaboración de tales diseños que orientarían el proceso andragógico de aprendizaje. Hoy en día, tanto en la Maestría en la Enseñanza de la Lectura y la Escritura en curso y, desde el reinicio de la X cohorte en Enero del 2014, se informó a quienes ingresaron que el proceso se caracterizaría por la ejecución de actividades presenciales y virtuales interactivas con encuentros cada 15 días para las asesorías. Así, de este modo, se estaría tomando ventaja de las aulas virtuales montadas en el sistema de gestión de cursos de distribución libre; es decir, el Entorno Modular de Aprendizaje Dinámico Orientado a Objetivos (MOODLE) de la Modalidad de Aprendizaje Dialógico Interactivo $(A D I)$ en la jornada anteriormente mencionada para emplear estrategias del enfoque dialógico interactivo.

Cabe destacar, que los participantes recibieron un curso introductorio que les permitió adiestrarse con el uso de la plataforma. No obstante, existe cierta incertidumbre con respecto a la efectividad de este proceso, por cuanto constituye una forma nueva de aprendizaje cuyo proceso inició en el año 2014. En el transcurso de este devenir, los participantes han mostrado preocupación por cuanto no todos los profesores han llevado a cabo una acción mixta que combine estrategias presenciales y virtuales, sino que muchos continúan con un proceso monótono tradicional en el que los facilitadores emplean más encuentros presenciales que en línea y cuyos participantes consideran poco conveniente, debido a que sus horarios laborales ameritan de la puesta en marcha del enfoque dialógico interactivo apoyado en las tecnologías de la información y la comunicación (TIC).

De este modo, cumplir a cabalidad con ambas responsabilidades. Del mismo modo, no existe una planificación específica por parte de muchos docentes que precise las actividades a abordar durante un semestre, debido a que a lo largo del proceso y sin contar con un consenso, el mismo modifica su cronograma, ocasionando así incomodidad a los participantes. 
Tal realidad se debe quizá a que existe poca supervisión del proceso de desarrollo del enfoque dialógico interactivo y el menoscabo en el seguimiento de la planificación instruccional de las unidades curriculares, orientada a acortar las brechas comunicacionales entre el docente y los estudiantes. Por otro lado, no existe fortalecimiento constante de la formación docente para el alcance de su desempeño óptimo dentro de un proceso dialógico interactivo apropiado.

La falta de fortalecimiento permanente puede conducir a la monotonía y a la práctica de una acción educativa netamente tradicional y puede conducir al desconocimiento del sistema de gestión de aprendizaje donde el docente debería ejercer su acción en la conformación de ambientes centrados en el estudiante, así como también, al desconocimiento de las necesidades instruccionales en la unidad curricular y falta de motivación docente en la implementación de la modalidad, puesto que no se estimula el uso de las tecnologías de información y comunicación a nivel docente.

En adición a lo planteado, se han presentado inconvenientes en cuanto a los procesos de comunicación síncrona y asíncrona por fallas en el sistema de conexión. Sin embargo, se han empleado otras páginas alternativas para evitar la interrupción de la interacción.

Considero oportuno agregar que a esta experiencia se suma la integración de la Maestría en Gerencia y Proyecto de Ingeniería, ya que uno de los profesores que participó en el curso de formación bajo el enfoque ADI, trabajó bajo esta visión a lo largo de un semestre en ese postgrado. Por esta y otras razones especificadas por los participantes de ese postgrado en relación a: la existencia de un sentir similar al de los participantes de la Maestría en la Enseñanza de la Lectura y la Escritura en cuanto a la preeminencia de un proceso monótono tradicional por parte de la mayoría de los profesores, la demanda de cambios conexos con los avances tecnológicos para facilitar el ajuste de horarios académicos y laborales de quienes forman parte del proceso andragógico, la necesidad de una comunicación ininterrumpida mediante el empleo de 


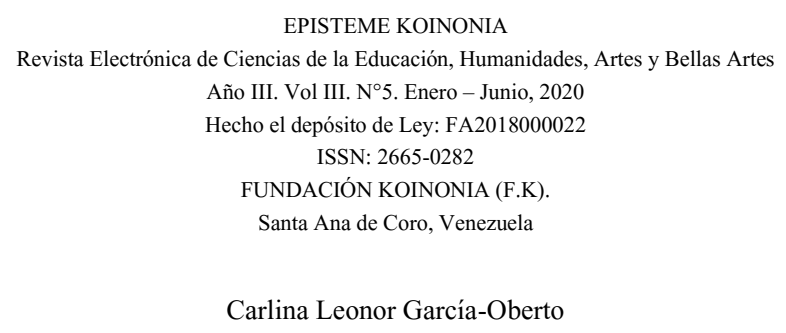

diversas herramientas y lo satisfactorio que fue la experiencia dialógica interactiva para todos los involucrados con uno de sus profesores, constituyeron motivos esenciales para estudiar esa vivencia singular.

Tomando como base las consideraciones previas, el presente estudio gira en torno a la generación de los fundamentos teóricos de la praxis andragógica en los estudios de postgrado bajo un enfoque dialógico interactivo desde una visión fenomenológicahermenéutica. Entendiéndose por praxis, de acuerdo con González (1997, p. 185), el proceso relacionado con los actos, la acción, la actividad y la actuación que se gesta dentro del contexto educativo. Dicho término, aunado con el concepto de Knowels (1975, p. 3), sobre Andragogía, implica un proceso en el que los sujetos autogestionan sus aprendizajes formulándose objetivos que los orientan a seleccionar e implementar las estrategias apropiadas tanto para el proceso didáctico como para su proceso evaluativo en colaboración con docentes, compañeros de estudio y otros recursos humanos y materiales en pro del alcance de una formación óptima.

La idea es comprender desde la visión del sujeto el contenido del sentido de esta realidad que en términos de Husserl, implica una exploración de la cosa misma, por medio de la concepción de un clima de comunicación horizontal con los informantes clave considerando la esencia respectiva de su conciencia, con sus respectivas formas de vivencias, praxis y correlatos de dicha praxis. Por tanto, los informantes clave (docente de postgrado que han sido partícipes de la Modalidad de Aprendizaje Dialógico Interactivo) serán los protagonistas del alcance de la trascendencia de su conciencia a través de la reflexión y la consideración de sus significaciones para llegar a la generación de un constructo teórico que aporte los elementos constituyentes de una praxis andragógica apropiada y efectiva en este nivel mediante la estimación del enfoque.

Vale resaltar que, no todos los docentes se muestran rehaceos a emplear este tipo de enfoque, ya que según conversaciones informales llevadas a cabo con los participantes 


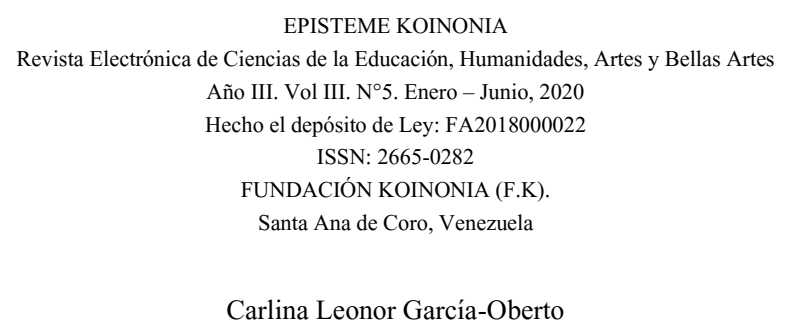

de la maestría, aunque son pocos los docentes que emplean el mismo, quienes lo han tomado en cuenta han propiciado un ambiente óptimo, interactivo, atractivo y socializador, que les ha permitido alternar fácilmente entre sus trabajos y su estudio, dando lugar así, a un aprendizaje productivo y significativo.

Por consiguiente, para comprender mejor este devenir y lo que puede aportar en el nivel andragógico, mediante la fenomenología trascendental, escudriño, de la mano de sus protagonistas (facilitadores de postgrado que han empleado el enfoque), las manifestaciones esenciales que me pueden ayudar a generar una teoría fundada en la interpretación de los significados asignados por el ser trascendental, tratando de llegar a una conciencia científica que se ajuste a lo dado en los estudios de postgrado bajo el enfoque dialógico interactivo; así, de esta manera, enjuiciar el fenómeno a partir del conocimiento intuitivo y la pureza del objeto, de quien aplica apropiadamente la dialogicidad y la interactividad.

Asimismo, se concibe la comprensión de la praxis docente desde su experiencia sobre la base de la aprehensión de los significados que trascienden la conciencia del yo y se interpreta el proceso educativo desde las reflexiones concebidas por Husserl (2013, p. 293-294) como noéticas (sentido del sujeto acerca del objeto dado como tal.) y noemáticas (objeto percibido como tal). Bajo esta concepción, se despliegan las interrogantes y luego los propósitos a los cuales se pretenden dar respuesta mediante el encuentro e interacción de la investigadora con los sujetos.

¿De qué forma concibe el sujeto el fenómeno puro de la praxis andragógica bajo el enfoque dialógico interactivo?

¿De qué manera se caracteriza el empleo apropiado del enfoque dialógico interactivo?

¿Qué reflexiones noéticas y noemáticas merece la esencia misma del enfoque dialógico interactivo? 


\section{Propósito general de la investigación}

Comprender la praxis andragógica en los estudios de postgrado desde una perspectiva teórica dialógica y fenomenológica Husserliana sobre la base de la aprehensión de los significados que trascienden la conciencia del yo.

\section{METODOLOGÍA DE LA INVESTIGACIÓN}

El método del presente estudio se fundamentó en la fenomenología trascendental de Husserl, aunado a un diseño emergente, el cual según Merlino (2009, p. 44) "se construye a medida que se avanza iterativamente en el proceso de investigación, yendo de los datos a los conceptos de la teoría social, de éstos a la reflexión y nuevamente a los datos". Todo ello implica que el conocimiento se construyó acudiendo a la lógica inductiva dirigiéndome a las partes, es decir a los facilitadores que fueron partícipes de la vivencia andragógica en los estudios de postgrado bajo el enfoque ADI para comprender su praxis desde su conciencia y así construir un todo sustentado en los datos aportados por los informantes clave de donde derivaron las categorías aportadas en atención a la realidad del ser.

Para tal fin, en sintonía con lo expresado por Merlino, mi intervención desde el punto de vista reflexivo condujo a la profundización del sentido de lo emergido a partir de la conciencia trascendental, lo cual, de la mano de los sujetos protagonistas del campo educativo estudiado pudo enriquecer la constitución del entramado teórico. A continuación resumo la vía metodológica a seguir con sus respectivos métodos, técnica y rigor científico.

\section{Proceso de generación de la teoría del contexto postgrado-ADI}

Rodríguez en Sandín, (2003, p. 139), indica que en la investigación cualitativa se generan las fases: preparatoria, de campo, analítica e informativa. En atención a dichas etapas, mi hilo conductor metodológico lo contextualicé en relación a la realidad 
estudiada con respecto a los estudios de postgrado bajo el enfoque de Aprendizaje Dialógico Interactivo (ADI). De este modo, la generación teórica se llevó a cabo tomando como base en la vivencia del ser trascendental.

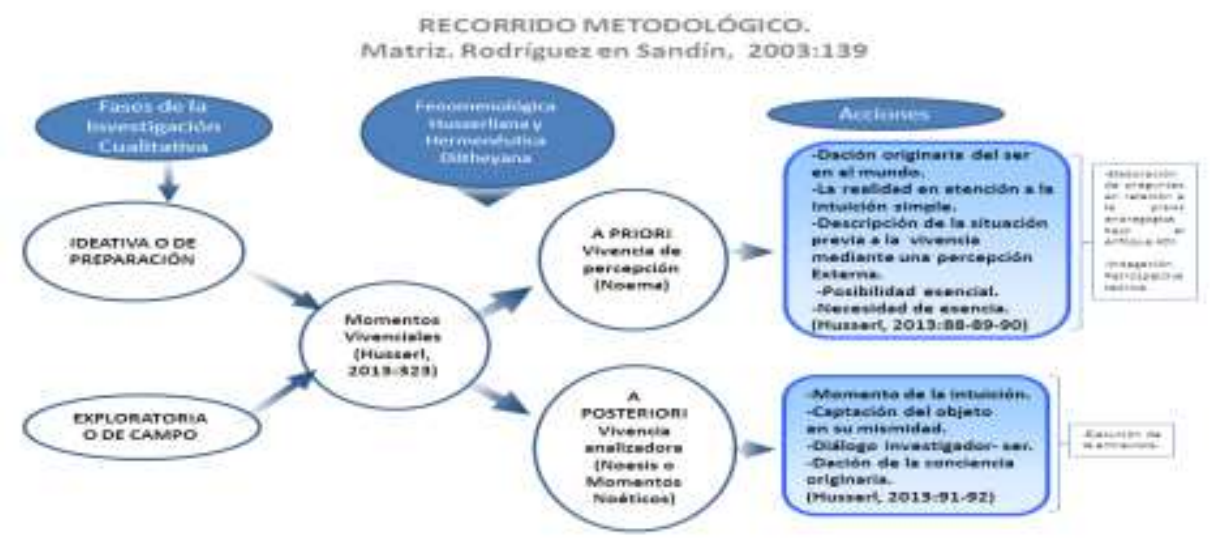

Figura 1. Fases de preparación y de campo. Fuente: Rodríguez en Sandín (2003, p. 139) adaptado por la autora, según concepciones de Husserl y Dilthey.

Tal como se puede concebir en la figura 1, muestro, en primera instancia, las fases ideativa y exploratoria que en correspondencia con la lógica de Husserl, se abordó la captación de la vivencia docente en los estudios de postgrado en un proceso dialógico interactivo, lo cual implicó la descripción esencial del objeto desde una concepción noética (subjetiva del ser.). En esta fase elaboré las preguntas en relación a la vivencia de los docentes en los estudios de postgrados bajo el enfoque de aprendizaje dialógico interactivo e hice una revisión teórica de las categorías de entrada.

\section{Técnicas e instrumentos para la recolección de la información}

Para este trabajo, seleccioné la entrevista a profundidad. Ésta según Piñero y Rivera (2012, p. 106), presume un "proceso en el que el investigador configura un ambiente distendido y de empatía para realizar preguntas o planteamientos abiertos que 


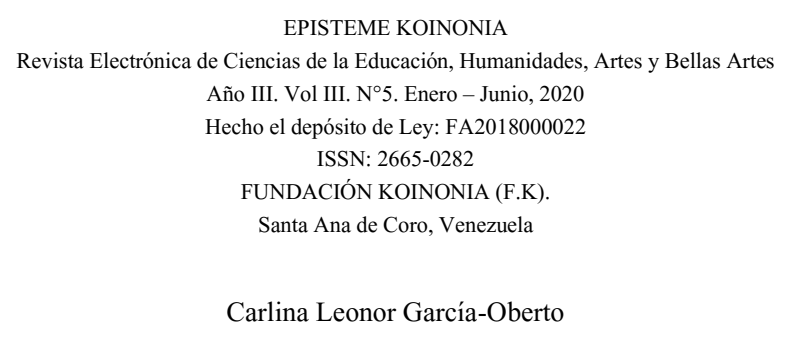

propicien por parte de los entrevistados, la expresión con sus propias palabras de su perspectiva personal sobre el tema".

Asimismo, Piñero y Rivera (2012: 106) exponen que esta técnica "es utilizada en forma combinada con otros procedimientos como la observación participante, los relatos de vida, testimonios focalizados, entre otros." Por consiguiente, empleé otra técnica que permitió conceder importancia tanto al lenguaje oral como a los elementos gestuales del sujeto; por ello acudí a la observación, la toma de notas y la transcripción como los diversos modos de complementar todos aquellos datos obtenidos por medio de la entrevista atendiendo, de este modo, a la relevancia de los gestos y las palabras según el criterio de Dilthey.

\section{Informantes clave}

Según Rojas, (2010: 66) la selección de los sujetos supone la selección de aquellos que puedan aportar información relevante para un estudio a profundidad. Debido a las indagaciones que en un principio realicé por medio de conversaciones informales con el coordinador de la Maestría en la Enseñanza de la Lectura y la Escritura y sus participantes, había seleccionado cinco informantes. Por consiguiente, los sujetos seleccionados estuvieron conformados por cinco (5) profesores quienes emplearon la plataforma ADI desde el inicio hasta la culminación de las Unidades Curriculares bajo su tutela.

\section{RESULTADOS}

Para conservar la confidencialidad de los informantes acudí al código de ética; y para ello, asigné pseudónimos a cada uno otorgándoles los siguientes: L-01, K-02, Y-03, D04 y E-05; de este modo, mis informantes y yo reconocemos de quién se trata cuando sus opiniones son mencionadas en el estudio. 
El procedimiento empleado para la sistematización teórica consistió en presentar primeramente, la esencia de la vivencia de cada sujeto desde su singularidad y su perspectiva descriptiva: para ello, muestro las categorías emergentes y la definición que cada cual aporta acerca de las mismas. En torno a lo anterior, cito lo expresado por el sujeto en su pureza, considerando la epojé, lo cual me facilita revelar la concepción de la conciencia acerca del noema.

\section{Esquema teorético: Síntesis interpretativa de la Andragogía innovadora trascendental de vida.}

Para Teppa (2012, p. 94), construir teorías significa "reducir los datos originales de muchos actores sociales y observaciones particulares a conceptos y convertirlos en un conjunto de afirmaciones de relación que puedan explicar y predecir sucesos y fenómenos". En el caso particular de este estudio doctoral, procedo a integrar todos los elementos emergidos que me permitieron redefinir la realidad estudiada bajo una concepción innovadora.

De igual modo, Teppa (2012, p. 99) precisa que las categorías definidas se integran en una síntesis conjetural explicativa para extender y darle densidad a la teoría. Por tal motivo, a continuación presento una matriz sintética que denomino "Esquema teorético: Síntesis de la teoría emergente" donde integro las manifestaciones y submanifestaciones constituyentes derivadas de las vivencias, las cuales dan lugar a la generación de una conceptualización propia fundamentada en lo dado por los sujetos según sus vivencias andragógicas bajo el enfoque ADI. Por ende, cada manifestación es definida según lo emergido como manifestación constituyente e hyle predicativo, es decir según las características dadas por los informantes acerca de los componentes que conforman la praxis andragógica sumado a mi interpretación sobre lo dado de forma judicativa. 
EPISTEME KOINONIA

Revista Electrónica de Ciencias de la Educación, Humanidades, Artes y Bellas Artes

Año III. Vol III. N ${ }^{\circ}$. Enero - Junio, 2020

Hecho el depósito de Ley: FA2018000022

ISSN: 2665-0282

FUNDACIÓN KOINONIA (F.K)

Santa Ana de Coro, Venezuela

Carlina Leonor García-Oberto

\section{Cuadro 1}

Síntesis interpretativa de la andragogía innovadora trascendental de vida.

옳

Concepto

옹

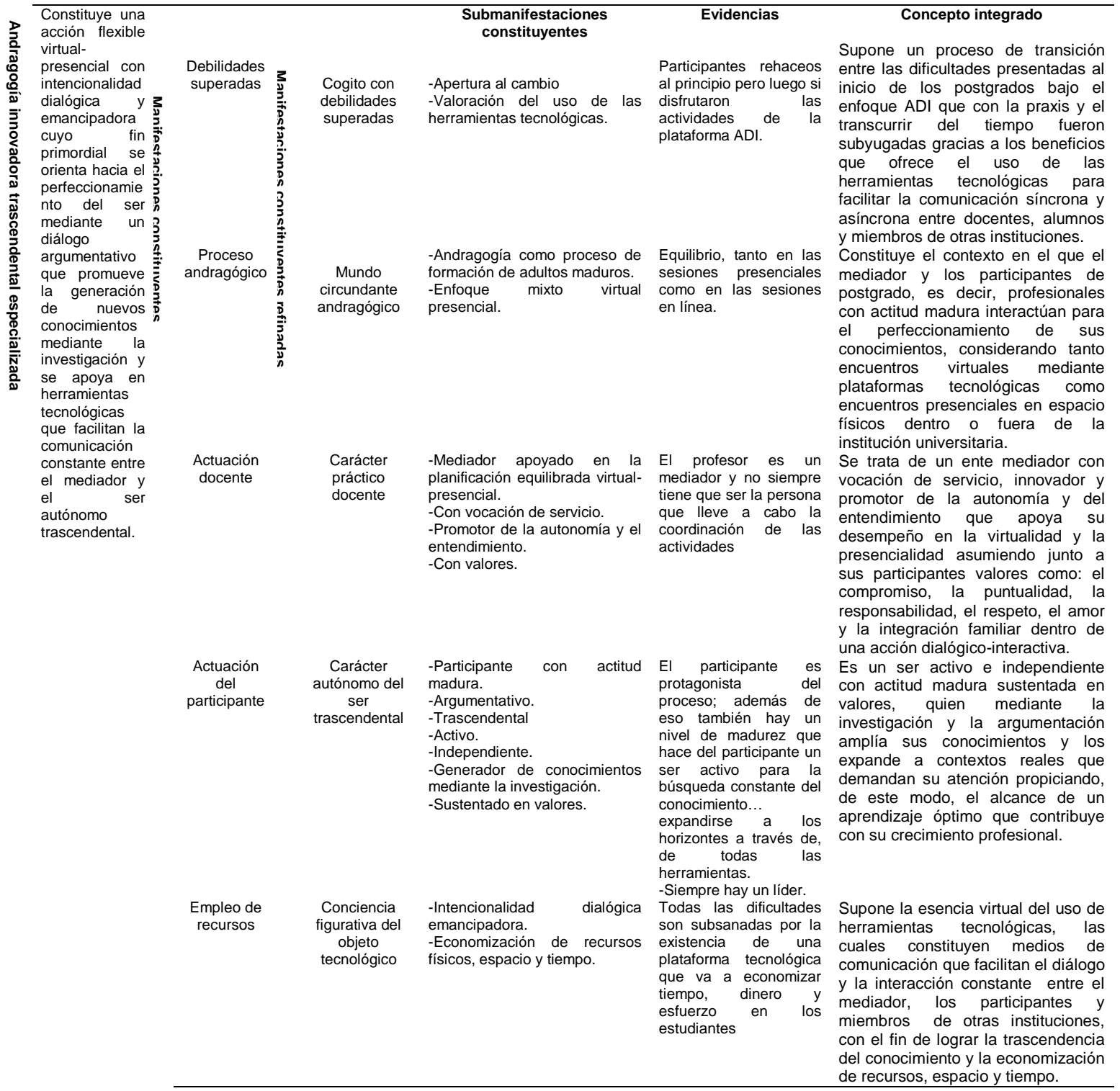

Fuente: Elaboración propia. 
Para construir una síntesis teórica interpretativa de la vivencia estudiada, conviene contrastar lo emergente con las teorías base de esta investigación. En tal sentido, Knowels (1980, p. 43), define la andragogía como un modelo asumido dentro de un proceso de maduración fundamentado en un aprendizaje autodirigido que en ocasiones esporádicas requiere de dependencia. Dentro de esta concepción, Knowels (1975, p. 2) concede importancia al aprendizaje autodirigido del alumno maduro, puesto que el mismo es capaz de promocionar la autogestión dentro de un clima de respeto mutuo, de diálogo y de participación activa con el docente y otros, donde se realizan proyectos de aprendizaje, de investigación, entre otros, fundamentados en la vida real.

En el caso particular de este tratado doctoral, emergió una andragogía que coincide con los postulados de Knowels, en el que el participante es autónomo, asume una actitud madura y participa activamente mediante la interacción con el facilitador, los compañeros de clase y los miembros de otras instituciones. No obstante, como otro aspecto particular precisado en la vivencia andragógica de los sujetos entrevistados, se destaca además, un proceso sustentado en el uso de herramientas tecnológicas que suscitan la superación de distintas barreras físicas y virtuales y promueven la comunicación permanente entre los involucrados, atendiendo así a distintas especialidades para la consolidación de los conocimientos previamente adquiridos en el nivel de pregrado. A ello se debe, la inclusión de los adjetivos que la identifican como innovadora, trascendental y especializada.

Por tal razón, el concepto integrado gira en torno a una acción flexible virtual-presencial con intencionalidad dialógica y emancipadora cuyo fin primordial se orienta hacia el perfeccionamiento del ser mediante un diálogo argumentativo que promueve la generación de nuevos conocimientos mediante la investigación y se apoya en herramientas tecnológicas que facilitan la comunicación constante entre el mediador y el ser autónomo trascendental. Bajo esta noción, se evidencia una diferencia singular con respecto a las consideraciones de Knowels, quien funda las bases de una 


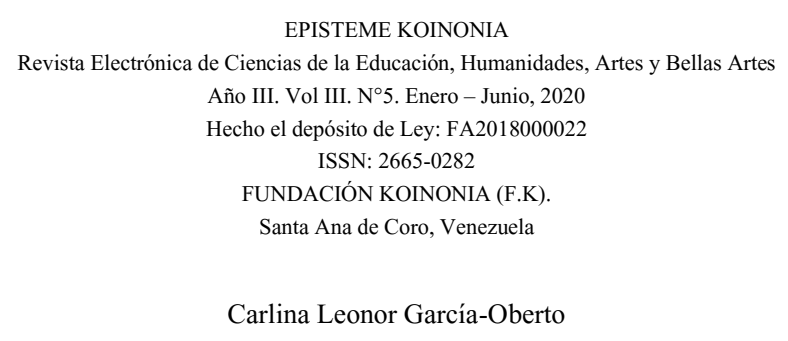

formación de adultos novedosa y significativa para la época, sin embargo, en el caso de este contexto educativo, se apoya en herramientas tecnológicas que, hoy en día, dan lugar a la comunicación síncrona y asíncrona no únicamente presencial sino también virtual.

Esta teoría emergente comprende la integración de cinco manifestaciones que integran y configuran la vivencia de los seres en postgrado, las cuales al ser refinadas recibieron denominaciones como: cogito con debilidades superadas, mundo circundante andragógico, carácter práctico docente, carácter autónomo del ser trascendental y conciencia figurativa del objeto tecnológico.

Al respecto, una vez germinado el cogito con debilidades superadas, fue concebido como un aspecto relevante y transitivo, ya que emanó como un proceso de atenuación de las barreras que los participantes generaron al principio de la práctica andragógica en relación al uso de herramientas tecnológicas. Posterior a ello, gracias al estímulo que recibieron los mismos de parte del mediador, tal avance fue aceptado. Por consiguiente, el ser describió una experiencia particular ejemplar donde tanto los seres en formación como el facilitador se ajustaron a un proceso nuevo para ellos pero, a su vez, significativo, puesto que estuvo apoyado en actividades en línea que facilitaron el desarrollo de una comunicación continua entre ellos.

Del mismo modo, la andragogía circunscrita en esta vivencia está constituida por un mundo circundante andragógico, el cual comprende la praxis andragógica como tal pero modelada de forma refinada en base a mi hilo conductor Husserliano en consonancia con la realidad explorada. De esta manera, germina una acción interactiva entre el mediador y los participantes de postgrado, quienes considerando tanto encuentros cara a cara como encuentros virtuales, se desenvuelven de forma óptima para lograr el refuerzo de sus conocimientos dentro de las distintas especialidades en las que cada uno se destaca. 
Lo anterior es cónsono con lo expuesto por Flecha (2007, p. 1), quien expresa que el proceso educativo no se vivencia únicamente en el aula, puesto que éste supera la barrera de las cuatro paredes encauzándose hacia una formación abierta y flexible que trasciende un proceso de comunicación permanente. También manifiesta que las tecnologías de la información y la comunicación (TIC) cumplen un rol importante en los procesos de formación de los individuos, puesto que instituyen un complemento de las actividades presenciales.

En el caso de los estudios de postgrados explorados en esta tesis, se hace énfasis en la planificación equilibrada virtual-presencial, es decir, se precisa la importancia de aplicar $50 \%$ de actividades presenciales y $50 \%$ de actividades en línea, debido a que de esta forma, se podrían alcanzar mejores resultados y mayor motivación del participante a ser parte de un proceso que no interrumpe su devenir formativo sino que facilita la entrega oportuna, flexible y responsable de sus actividades pendientes. A pesar de que se considera tal aseveración como una posibilidad ideal, los informantes afirmaron la dificultad de lograrlo a cabalidad, por cuanto, según sus vivencias, hubo varias situaciones que impidieron su cumplimiento, sin embargo, se establece como una de las condiciones propicias para el alcance de una praxis andragógica innovadora.

Por otro lado, la teoría emergente se configura con el carácter práctico del docente como ente mediador y promotor de la comunicación horizontal, cuyo desempeño se sustenta en la vocación de servicio. En este particular, Flecha (2007, p. 3), establece que la sociedad informacional tiene una base igualitaria de formación de opciones semejantes a todas las personas con independencia de su clase social o de su cultura. De este modo, su consideración propende a una interacción equitativa mediada por las tecnologías, tal como lo exponen los informantes. A esto se suma, la vocación como sub manifestación clave de la andragogía descrita en este acontecer específico, por cuanto el mediador asume un rol activo que no se rinde ante las dificultades sino que 
escudriña todas las vías y opciones posibles para compartir y fortificar los conocimientos a adquirir en los estudios de cuarto nivel.

Asimismo, el mediador es innovador y se mantiene a la par con los cambios a fin de emplear las herramientas posibles que promuevan la autonomía y del entendimiento entre los involucrados. Por ende, apoya su desempeño en la virtualidad y la presencialidad asumiendo junto a los participantes valores como: el compromiso, la puntualidad, la responsabilidad, el respeto, el amor y la integración familiar. En relación a lo emergido, conviene mencionar como aspecto puntual el surgimiento de los valores como el amor y la integración familiar, aspectos característicos novedosos propios de esta realidad que nace de un ambiente armónico vivenciado no sólo entre docentes y estudiantes sino también con el personal administrativo y obrero de la universidad. Por lo tanto, la andragogía aquí gestada va más allá de un aula de clase constituyéndose así en un sistema abierto, por cuanto no sólo implica un hecho educativo sino también un acontecer familiar.

En cuanto a otra de las manifestaciones, derivó el carácter autónomo del ser trascendental, denominado así a partir del refinamiento del término "actuación del participante", quien asume un rol activo e independiente y una actitud madura sustentada en valores. Por consiguiente, es un sujeto capaz de participar en diálogos argumentativos ampliando sus conocimientos mediante la investigación. Además, emplea sus habilidades intelectuales en contextos reales para alcanzar el aprendizaje óptimo de nuevas generalidades. Es menester resaltar que la argumentación según Ríos (2007, p. 231), reside "en la expresión de un razonamiento mediante el cual se intenta probar o refutar una tesis". En materia de esta experiencia, ésta radica tanto en la expresión del acuerdo y desacuerdo acerca de un tema como en la indagación de teorías que fundamentan las opiniones propias de quien manifiesta una declaración, a fin de llegar un consenso resultante del diálogo, la dialéctica, la interacción y la retroalimentación. 
Por esta concepción, una de las novedades precisadas en esta vivencia andragógica consiste en el desempeño del estudiante como ser trascendental, puesto que el mismo no se conforma con la asimilación de una noción teórica sino que acude a lo práctico insistiendo en la generación de nuevas perspectivas fundamentadas en la realidad. Igualmente, supera las cuatro paredes de un aula de clase, ya que comparte saberes con miembros de otras instituciones y comunidades. Sobre la base de lo expuesto, el participante es un líder que puede tomar iniciativas propias y se relaciona de forma ecuánime con el facilitador para la toma de decisiones consensuadas a lo largo de la praxis andragógica. Inclusive, todos tienen la misma oportunidad de ejercer un liderazgo, por cuanto participan en actividades grupales que los conducen a jugar roles rotativos, es decir, roles de coordinadores, secretarios, entre otros que pueden ser asumidos por uno en una oportunidad y por otros en otra actividad.

La andragogía emergida también se configura con la conciencia figurativa del objeto tecnológico, proposición refinada para matizar la teoría y hacer referencia al empleo de recursos. Esta se instituye como la esencia virtual del uso de herramientas tecnológicas que facilitan el diálogo y la interacción constante entre el mediador, los participantes y los miembros de otras instituciones, de este modo, facilita el alcance de la trascendencia del conocimiento y la economización de recursos, espacio y tiempo.

En relación a las herramientas tecnológicas y las estrategias existen diversas definiciones pero si se trata de establecer una diferencia entre ellas, conviene citar a autores como Arias y otros (2012, p. 22), quienes señalan que las herramientas son aplicadas por el docente, con el fin de emplear estrategias para el desarrollo del proceso pedagógico. Por su parte, Esteban y Zapata (2008, p. 1) refieren que las estrategias constituyen "un plan de acción ante una tarea que requiere una actividad cognitiva que implica aprendizaje". Luego expresan que éste "implica habilidades y destrezas que el aprendiz ha de poseer previamente y una serie de técnicas que se aplican en función de las tareas a desarrollar". A esto adicionan que "ha de existir 
conciencia de: a) la situación sobre la que se ha de operar (problema a resolver, datos a analizar, conceptos a relacionar, información a retener, etc.)".

De allí, resulta claro que el mundo andragógico de esta experiencia singular se concibe como innovadora, debido a que cuenta con el empleo de una serie de herramientas virtuales como: libros digitalizados, archivos PDF, documentos Word, plataforma Moodle, entre otras que complementan o sustituyen los recursos físicos. De igual forma, tiene como característica particular el empleo de herramientas dentro de dicha plataforma, tales como: wiki, blogs, foro, chats, entre otras; así como de páginas externas como: redes sociales, páginas web, correo, foro, blogs, videos; los cuales pasan a ser estrategias cuando el mediador y los estudiantes comprenden su utilidad operativa, de esta manera, se pueden aplicar: interacciones presenciales mediante videoconferencias, discusiones productivas mediante foro, debate a través del chat, incluyendo diversidad de acciones que se ajusten a los contenidos por aprender 0 fortalecer.

En términos generales, la teoría emergente resurge como una flamante andragogía capaz de dar respuesta a las demandas educativas de hoy en día en contextos diversos que requieren de la integración de un enfoque mixto (virtual-presencial) para el impulso de una formación óptima y el perfeccionamiento profesional. Luego de exponer la síntesis teorética redefinida según la vivencia docente, procedo a desglosar las reflexiones que reúnen y destacan la relevancia de este acontecer investigativo.

\section{REFLEXIONES DE CIERRE}

\section{Significado de las partes y el todo}

Desde mi visión, me planteo la siguiente interrogante: ¿cómo se evidenció la integración de las partes y el todo dentro de la praxis andragógica? De principio, cito a mis guías fenomenológico y hermenéutico. En virtud de sus pensamientos, Husserl (2013), afirma que las singularidades reducidas constituyen manifestaciones clave de lo 
trascendido en la conciencia acerca de la vivencia, mientras que Dilthey (2000), supone que el significado del todo requiere el sentido de sus partes y sus partes el significado del todo. De este modo se evidencia la integración noético - noemática de las reducciones crítica, eidética y trascendental del juicio de cada individuo, en el que eliminé todo perjuicio y me centré en lo dado según la vivencia descrita.

Luego, tal como se precisó en el apartado anterior, para reducir la gran cantidad de datos emergidos, acudí a la "esencia de la trascendencia", por cuanto reuní con mayor especificidad y simplicidad lo aportado por el sujeto, resaltando, de este modo, los atributos relevantes de la vivencia. De esta manera, tanto la categoría y subcategorías que trascendieron derivaron de la esencia judicativa propiciada desde la conciencia del ser en relación a su experiencia. Ahora bien, al unir estas partes y convertirlas en un todo derivaron manifestaciones que caractericé como constituyentes.

Con respecto a la praxis andragógica bajo el enfoque $A D I$, los docentes se apoyan en una planificación equilibrada, es decir, 50\% presencial y 50\% virtual, no obstante, no se logró a cabalidad por cuanto mientras un profesor, por ejemplo, L-01 alcanzó mayor comunicación presencial, la otra docente cumplió mayor virtualidad. A pesar de ello, todos consideran que lo ideal es fundamentar la acción en el equilibrio. Uno de los términos mencionado únicamente por D-04 ha sido la palabra metodología para definir la experiencia andragógica, sin embargo, este informante al igual que las demás concibe esta etapa educativa como un proceso de orientación que facilita el aprendizaje mediante el aprovechamiento de herramientas tecnológicas.

Dentro de estas concepciones, cabe mencionar que la informante L-01, hizo referencia a la importancia del conocimiento científico, profundo y argumentativo de los participantes coincidiendo con las opiniones del resto de los sujetos estudiados. Según su apreciación personal, éste es necesario en ese nivel tanto en la modalidad tradicional como en la mixta. Asimismo, los docentes acuerdan en el hecho de considerar al estudiante como un ente activo, con disposición a llevar sus 
conocimientos a un nivel mucho más amplio del que ya poseen por medio del intercambio de saberes. Asimismo, Y-03, D-04 y E-05 resaltaron la autonomía del aprendiz como punto importante en estudios de cuarto nivel.

También las informantes Y-03, D-04 y E-05 afirmaron que la madurez constituye una propiedad relevante dentro de los estudios de postgrado, por cuanto el estudiante asume un rol independentista mediante el manejo de herramientas que lo conducen a demostrar los conocimientos adquiridos a través de participaciones protagónicas, activas y constructivas mediadas por un facilitador que, de forma horizontal, orienta el aprendizaje.

Otro aspecto concordante a señalar entre lo expuesto por todos los informantes para la unión de las partes, lo constituyó la valoración del uso de las herramientas tecnológicas no sólo para la formación de los estudiantes de la universidad sino para lograr la trascendencia en el intercambio de saberes con integrantes de otras universidades; de este modo, se logró alcanzar la vinculación de diversas vivencias y el enriquecimiento del conocimiento científico. En cuanto a las herramientas empleadas, todos han asegurado emplear foro, wiki y chat de la plataforma Moodle y otros enlaces externos.

Desde otro ángulo, es propicio señalar que entre las debilidades mencionadas por algunos profesores se precisan: resistencia al uso de estrategias tecnológicas por parte de los docentes y los estudiantes, situación que poco a poco fue superada con la dedicación, orientación y acompañamiento constante de los facilitadores. Del mismo modo, emergió el abandono del diálogo pero con intencionalidad de los involucrados a rescatarlo; esto se lograría mediante esfuerzos realizados no sólo por parte del personal docente sino también por coordinadores, administrativos y obreros en el contexto educativo.

A manera de conclusión, las conciencias trascendentales contribuyeron a la generación de manifestaciones y sub manifestaciones constituyentes sustentadas en una acción andragógica donde el diálogo y la interacción social aparte de promover la participación 


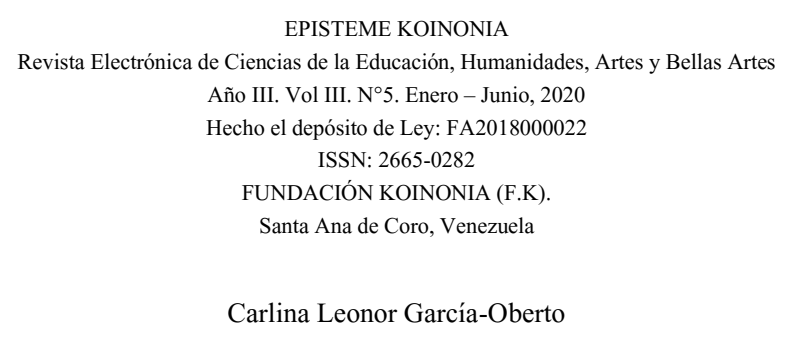

activa de los involucrados, permitió la superación de las debilidades que los mismos poseían al inicio de sus vivencias.

Apreciando las diversas realidades descritas, considero relevante citar a Morse (2003, p. 164), cuando expone que "la fenomenología de Husserl estudia la experiencia para revelar la conciencia." De este modo, todas las vivencias han sido exploradas desde la conciencia del ser trascendental quien desde su singularidad configuró la esencia de su vivencia atendiendo al momento y lugar particular en el que ocurrió su realidad; por ende, para comprender esta realidad, tomé también como base el aporte de Dilthey quien precisa lo siguiente: toda vivencia singular está referida a un yo que articula todas las partes de la vivencia para formar una estructura lógica resultante de la percepción del ser que teje una representación resultante de la realidad dinámica cambiante.

Por consiguiente, considerando la integración de las vivencias de cada informante en momentos distintos, las teorías base y mi interpretación sobre cada vivencia, procedí a la construcción de la teoría a partir de definiciones, conceptualizaciones, contrastaciones y nexos de las categorías y subcategorías emergidas. Tales hallazgos fueron luego interrelacionados y contrastados tomando en cuenta los aportes a profundidad de la literatura teórica consultada para presentar los aportes refinados derivados de cada uno de los captos interpretados.

En consonancia con la epojé, se develaron realidades cósicas del ser trascendental en su pureza, la cual radicó en la orientación que mi hilo conductor Husserliano me otorgó, ya que me desligué de todo prejuicio, concediendo libertad al sujeto para expresar sus vivencias y "mostrarse a sí mismo" tal como lo expresa Morse (2003, p. 141), de esta forma, surgieron diversas evidencias de la conciencia que dieron lugar al nacimiento de una teoría emergente propia de lo dado acerca de los estudios de postgrado bajo un enfoque mixto.

Como resultado, se evidenció la manera en la que el sujeto reveló su conciencia desde lo intuido en su vivencia desde la intencionalidad, la temporalidad, la intersubjetividad, 


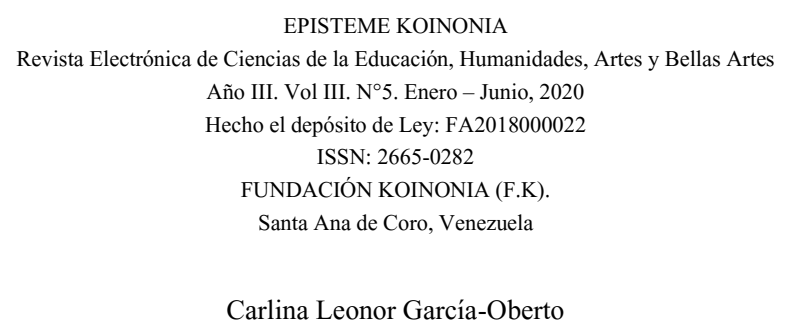

lo cual lo condujo a describir una realidad innovadora que dio lugar a la generación teórica representada en un cardón categórico trascendental de vida y redefinida en un esquema teorético sustentado en la andragogía innovadora trascendental especializada.

\section{FINANCIAMIENTO}

No monetario

\section{AGRADECIMIENTOS}

Un agradecimiento especial a mi tutor y a todos los docentes e investigadores que me apoyaron en el desarrollo de la investigación; en especial a los informantes claves por su tiempo y dedicación.

\section{REFERENCIAS}

Arias, M., Sandia B. y Mora, E. (2012). La didáctica y las herramientas tecnológicas web en la educación interactiva a distancia. [Didactics and technological web tools in interactive distance education]. EDUCERE. Artículos arbitrados. Año 16, № 53. Mérida, Venezuela.

Dilthey, W. (2000). Dos escritos sobre hermenéutica. [Two writings on hermeneutics]. Ediciones Istmo, S.A. Madrid.

Flecha, R. (2007). La educación en la Sociedad de la Información. [Education in the Information Society]. Recuperado a partir de https://n9.cl/9eo3

González, A. (1997). Estructuras de la praxis. [Structures of praxis]. Editorial Trotta, S.A. Madrid. Editorial Trotta, S.A. Madrid.

Husserl, E. (1949). Ideas relativas a una fenomenología pura y una filosofía fenomenológica. [Ideas regarding a pure phenomenology and a phenomenological philosophy]. Fondo de cultura económica. México, D.F. 


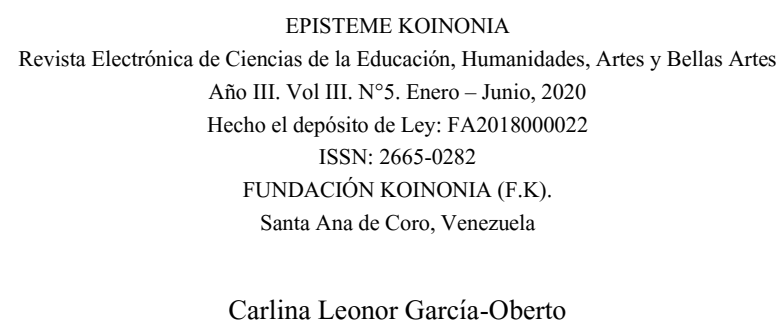

Husserl, E. (2002). Lecciones de fenomenología de la conciencia interna del tiempo. [Lessons in phenomenology of the internal consciousness of time]. Editorial Tortta, S.A. Madrid.

Husserl, E. (2013). Ideas relativas a una fenomenología pura y una filosofía fenomenológica. [Ideas regarding a pure phenomenology and a phenomenological philosophy]. Fondo de cultura económica. México, D.F.

Knowels, M. (1975) Self-Directed Learning: A Guide for Learners and Teachers, N.Y.: Cambridge Book Company. Extracto y traducción del libro original.

Knowles, M. (1980). The moderm practice of adult education. From pedagogy to andragogy. Cambridge. The adult education company. New York.

Merlino, A. (2009). Investigación cualitativa en ciencias sociales temas, problemas y aplicaciones. [Qualitative research in social sciences themes, problems and applications.]. Artes Gráficas Buschi S.A. Argentina.

Morse, J. (2003). Asuntos críticos en los métodos de investigación cualitativa. [critical in qualitative research methods issues]. Editorial Universidad de Antioquia. Colombia.

Piñero, M. y Rivera, M. (2012). Investigación cualitativa: orientaciones procedimentales. [Qualitative research: procedural guidelines] UPEL-IPB. Barquisimeto.

Ríos, J. (2007). Epistemología. Fundamentos generales. [Epistemology. General foundations]. Universidad Santo Tomás. Santafé de Bogotá D.C.

Rojas, B. (2010). Investigación cualitativa. Fundamentos y praxis. [Epistemology. General foundations]. Edición FEDUPEL. Caracas.

Sandín, E. (2003). Investigación cualitativa en educación. Fundamentos y tradiciones. [Epistemology. General foundations]. 1era. Edición. McGraw-Hill/ Interamericana. Madrid. España.

Teppa, S. (2012). Análisis de la información cualitativa y construcción de teoría. [Qualitative information analysis and theory construction]. Primera edición Gema, C. A. Venezuela. 